\title{
Protein subcellular localization prediction of eukaryotes using a
} knowledge-based approach

\author{
Hsin-Nan Lin ${ }^{1,2,3}$, Ching-Tai Chen ${ }^{1,2,3}$, Ting-Yi Sung ${ }^{2}$, Shinn-Ying $\mathrm{Ho}^{3}$ \\ and Wen-Lian $\mathrm{Hsu}^{* 2}$
}

\author{
Addresses: ${ }^{1}$ Bioinformatics Program, Taiwan International Graduate Program, Academia Sinica, Taipei, Taiwan, Republic of China, \\ ${ }^{2}$ Bioinformatics Lab., Institute of Information Science, Academia Sinica, Taipei, Taiwan, Republic of China and ${ }^{3}$ Institute of Bioinformatics, \\ National Chiao Tung University, Hsinchu, Taiwan, Republic of China \\ E-mail: Hsin-Nan Lin - arith@iis.sinica.edu.tw; Ching-Tai Chen - caster@iis.sinica.edu.tw; Ting-Yi Sung - tsung@iis.sinica.edu.tw; \\ Shinn-Ying Ho - syho@mail.nctu.edu.tw; Wen-Lian Hsu* - hsu@iis.sinica.edu.tw \\ *Corresponding author
}

from Asia Pacific Bioinformatics Network (APBioNet) Eighth International Conference on Bioinformatics (InCoB2009) Singapore 7-II September 2009

Published: 3 December 2009

BMC Bioinformatics 2009, 10(Suppl I5):S8 doi: 10.1186/147I-2105-10-SI5-S8

This article is available from: http://www.biomedcentral.com/I47I-2105/I0/SI5/S8

(c) 2009 Lin et al; licensee BioMed Central Ltd.

This is an open access article distributed under the terms of the Creative Commons Attribution License (http://creativecommons.org/licenses/by/2.0), which permits unrestricted use, distribution, and reproduction in any medium, provided the original work is properly cited.

\begin{abstract}
Background: The study of protein subcellular localization (PSL) is important for elucidating protein functions involved in various cellular processes. However, determining the localization sites of a protein through wet-lab experiments can be time-consuming and labor-intensive. Thus, computational approaches become highly desirable. Most of the PSL prediction systems are established for single-localized proteins. However, a significant number of eukaryotic proteins are known to be localized into multiple subcellular organelles. Many studies have shown that proteins may simultaneously locate or move between different cellular compartments and be involved in different biological processes with different roles.

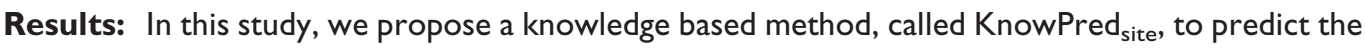
localization site(s) of both single-localized and multi-localized proteins. Based on the local similarity, we can identify the "related sequences" for prediction. We construct a knowledge base to record the possible sequence variations for protein sequences. When predicting the localization annotation of a query protein, we search against the knowledge base and used a scoring mechanism to determine the predicted sites. We downloaded the dataset from ngLOC, which consisted of ten distinct subcellular organelles from 1923 species, and performed ten-fold cross

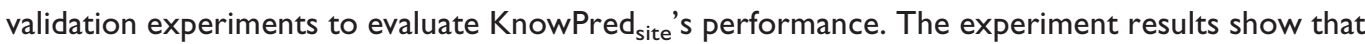
KnowPred $_{\text {site }}$ achieves higher prediction accuracy than ngLOC and Blast-hit method. For singlelocalized proteins, the overall accuracy of KnowPred ${ }_{\text {site }}$ is $91.7 \%$. For multi-localized proteins, the overall accuracy of KnowPred site is $72.1 \%$, which is significantly higher than that of ngLOC by 12.4\%. Notably, half of the proteins in the dataset that cannot find any Blast hit sequence above a specified threshold can still be correctly predicted by KnowPred site. $_{\text {. }}$.
\end{abstract}




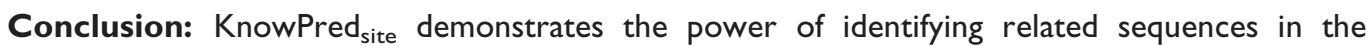
knowledge base. The experiment results show that even though the sequence similarity is low, the local similarity is effective for prediction. Experiment results show that $\mathrm{KnowPred}_{\text {site }}$ is a highly accurate prediction method for both single- and multi-localized proteins. It is worth-mentioning

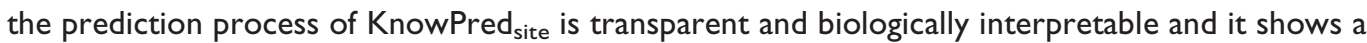
set of template sequences to generate the prediction result. The KnowPred site $_{\text {prediction server is }}$ available at http://bio-cluster.iis.sinica.edu.tw/kbloc/.

\section{Background}

Protein subcellular localization (PSL) is important to elucidate protein functions as proteins cooperate towards a common function in the same subcellular compartment [1]. It is also essential to annotate genomes, to design proteomics experiments, and to identify potential diagnostic, drug and vaccine targets [2]. Determining the localization sites of a protein through experiments can be time-consuming and labor-intensive. With the large number of sequences that continue to emerge from the genome sequencing projects, computational methods for protein subcellular localization at a proteome scale become increasingly important.

Most existing PSL predictors are based on machine learning algorithms. They can be categorized by the feature sets used for building prediction models. A group of methods use features derived from primary sequence [3-7]; some utilize various biological features extracted from literature or public databases [2,8-13]. Other features are also used in different methods, e.g., phylogenetic profiling [14], domain projection [15], sequence homology [5], and compartment-specific features [16].

A simple and reliable way to predict localization site is to inherit subcellular localization from homologous proteins. Therefore, in [5] a hybrid method was proposed, which combined an SVM based method with a sequence comparison tool to find homology to improve the performance. However, some homologous proteins are not similar in sequences, but in structures. For example, the sequence identity between proteins $1 a a b$ and $1 j 46$ is only $16.7 \%$ but they are structurally homologous and classified into the same family (HMG-box) in the SCOP classification. For such cases, it is difficult to discover the homologous relationship using sequence comparison methods. Profile-profile alignment methods [17-21] are capable of identifying remote homology; nevertheless, they are relatively slow.

Most of the PSL prediction systems are established particularly for single-localized proteins. A significant number of eukaryotic proteins are, however, known to be localized into multiple subcellular organelles [22,23]. In fact, proteins may simultaneously locate or move between different cellular compartments and be involved in different biological processes with different roles. This type of proteins may take a high proportion, even more than 35\% [22]. In addition, the majority of existing computational methods have the following disadvantages [23]: 1) they only predict a limited number of locations; 2) they are limited to subsets of proteomes which contain signal peptide sequences or with prior structural/functional information; 3) the datasets used for training are for specific species, which is not sufficiently robust to represent the entire proteomes. Thus, most of the computational methods are not sufficient for proteome-wide prediction of PSL across various species.

Thus in this study, we propose a knowledge based approach, called KnowPred siter $_{\text {using local sequence }}$ similarity to find useful proteins as templates for site prediction of the query protein. It is designed to predict localization site(s) of single- and multi-localized proteins and is applicable to proteome-wide prediction. Furthermore, it only requires protein sequence information and no functional or structural information is required. Notably, prediction results can be explained by the template proteins which are used to vote for the localization sites. The Knowledge-based prediction scheme has been shown to be effective in predicting protein secondary structure [24,25] and local structure [26]. To evaluate our knowledge-based site prediction method, we used the ngLOC dataset [23] to perform tenfold cross validation to compare with existing methods. The dataset consists of ten subcellular proteomes from 1923 species with single- and multi-localized proteins. KnowPred $_{\text {site }}$ achieved $91.7 \%$ accuracy for singlelocalized proteins and $72.1 \%$ accuracy with both sites correctly predicted for multiple localized proteins.

\section{Methods}

The main idea behind KnowPred ${ }_{\text {site }}$

KnowPred $_{\text {site }}$ predicts PSL based on a knowledge base, which is constructed to capture local sequence similarity 
of two proteins even when they have sequence identity less than $25 \%$. However, such local similarity is difficult to be detected using the traditional alignment algorithm due to the low sequence similarity. Therefore we adopt the transitivity relationship, which was firstly used in [27] for clustering protein sequences, to capture local similarity between protein sequences. Transitivity refers to deducing a possible similarity between protein $A$ and protein $C$ from the existence of a third protein $B$, such that $A$ and $B$ as well as $B$ and $C$ are homologues if the sequence identity between $A$ and $B$ as well as that between $B$ and $C$ is above the predefined threshold. Figure 1(a) shows an example of transitivity relationship among protein $A$, protein $B$, and protein $C$. Protein $A$ and protein $B$ share sequence identity of $34 \%$, and protein $B$ and protein $C$ share sequence identity of $27 \%$, whereas protein $A$ and protein $C$ only share sequence identity of $12 \%$. Using the transitivity relationship, remote homologous relationship and local similarity between protein $A$ and protein $C$ can be detected.

In this paper, we apply the transitivity concept to peptide fragments instead of the protein sequences to obtain local similarities between remotely homologues. Protein $A$ and protein $C$ share local similarity if there is a peptide fragment similar (formal definition of peptide similarity will be discussed in next subsection) to subsequences in protein $A$ and protein $C$. Figure 1(b) illustrates the idea, in which protein $A$ and $C$ are aligned with protein $B 1$ and protein B2 (B1 and B2 can be identical, homologous or non-homologous). If there is a peptide fragment shared by both $B 1$ and $B 2$, the corresponding peptide fragments in protein $A$ and protein $C$ are inferred as locally similar

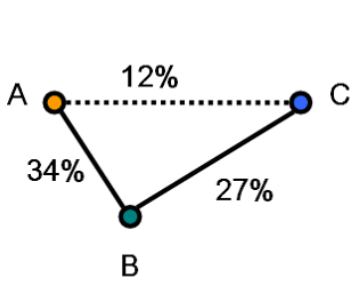

(a)

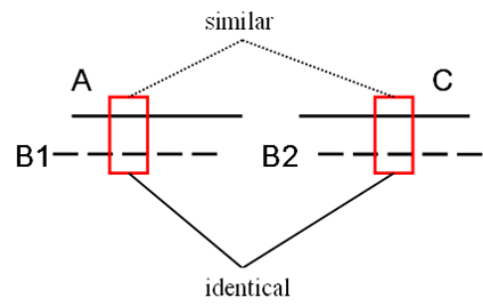

(b)
Figure I

Two different transitivity relationships. (a) Protein $A$ and protein $B$ share sequence identity of $34 \%$, and protein $B$ and protein $C$ share sequence identity of $27 \%$, whereas protein $A$ and protein $C$ only share sequence identity of $12 \%$. We infer the homologous relationship between $A$ and protein $C$ through protein $B$. (b) Protein $A$ and protein $C$ are aligned with protein $B I$ and protein $B 2$. The peptide fragments of $B I$ and $B 2$ besieged by the rectangles are identical, the two corresponding peptide fragments of $A$ and $C$ are considered to be similar. between protein $A$ and protein $C$. The shared peptide may represent a possible sequence variation in evolution. Moreover, if protein $A$ and protein $C$ are remotely homologous, there is likely more "shared" sequence fragments in different protein $B^{\prime}$ s to characterize their similarity. However, not all such proteins $A$ and $C$ which share local similarity are homologous. Some local similarities may arise without common ancestry. Short sequences may be similar by chance, and sequences may be similar because both are selected to bind to a particular protein. In order to avoid ambiguity, we define such proteins $A$ and $C$ which share local similarity as "related sequences".

\section{Construction of the knowledge base SPKB}

Given a dataset of proteins with known localization sites, we construct a knowledge base, called SimilarPeptide Knowledge Base (or SPKB in short). The dataset used to construct $S P K B$ will be described in the Result section. To construct the knowledge base, we first use the native sequence of each protein in the dataset to extract the fixed-length peptide fragments by using a sliding window of length $w$. Each peptide sequence as well as its protein source and the localization site information are stored in SPKB. Since the performance of knowledgebased methods relies on the size of the knowledge base, we then perform PSI-BLAST search with parameters $j=3$, $e=0.001$ on each protein in the dataset against the NCBInr database to find similar sequences. Since the NCBInr database contains only the protein sequence information, the localization annotation of peptides generated by similar sequences is determined as follows. Specifically, given a query protein sequence $q$, PSI-BLAST would generate a large number of significant local pairwise alignments called high-scoring segment pairs (HSPs) between $q$ and its similar proteins. An example of an HSP is shown in Figure 2. Statistically significant BLAST hits usually signify sequence homology. We assumed that in an HSP, the similar peptide sequences in the counterpart sequence (denoted by "Sbjct") represent the possible sequence variations to the corresponding peptide in the query (denoted by "Query"), i.e., the protein $q$. We use the same sliding window of length $w$ to generate all peptide fragments in each HSP. Two amino acids aligned together in an HSP are said to be interchangeable if they have a positive score in the BLOSUM62 (an interchangeable residue pair is represented as an amino acid letter or a plus symbol in an HSP). The number of amino acid pairs being interchangeable within a sliding window represents the similarity level of the two peptide fragments. A peptide in Sbjct is called a similar peptide if it has at least $k$ residues interchangeable to those of the corresponding peptide in Query. A similar peptide is used to signify local sequence 


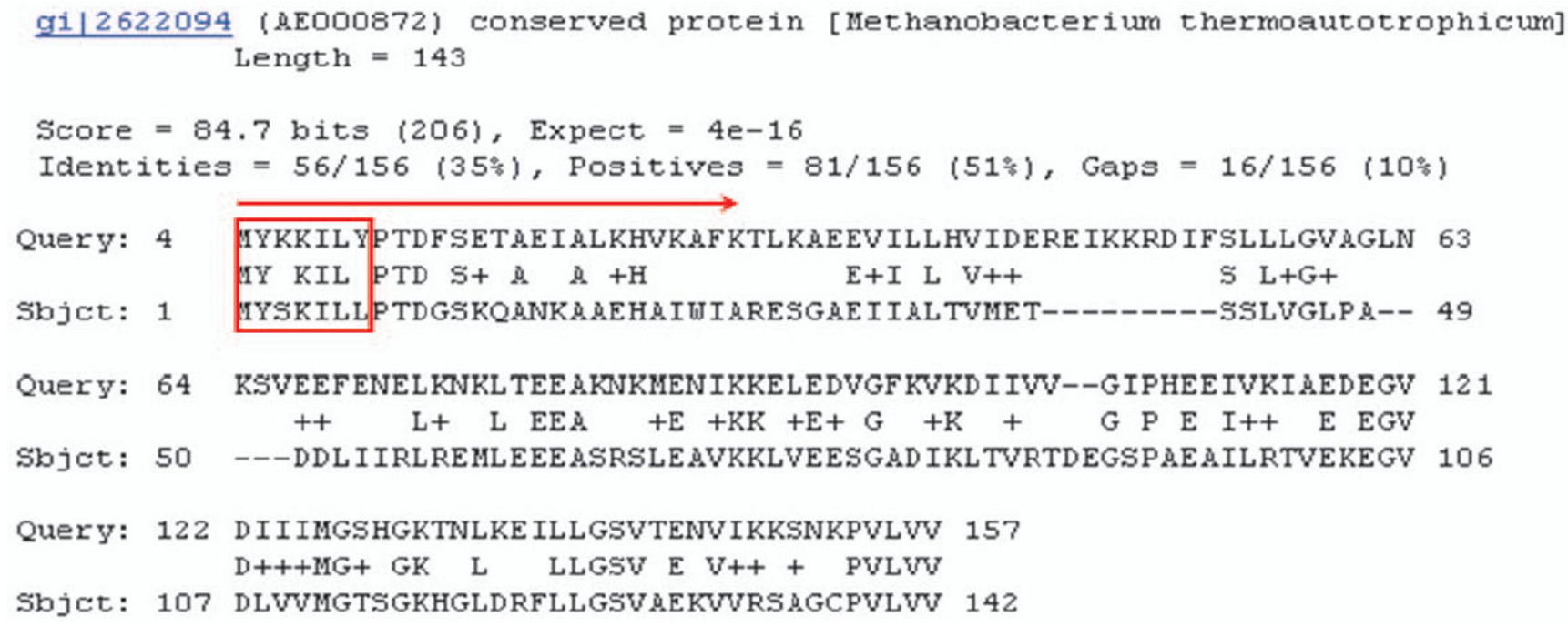

\section{Figure 2}

A real example of HSP found by PSI-BLAST. We define that MYSKILL (assuming that the window size is 7) is a similar peptide of MYKKILY and we treat it as an extended sequence feature of the query protein. The similarity level of MYSKILL and MYKKILY is 5 since there are five interchangeable residue pairs within that window. We can generate multiple similar peptides from protein gi|2622094 (Sbjct) for the query protein.

similarity between Sbjct and Query and thus is assigned the localization annotation of the protein $q$.

Performing PSI-BLAST search for all proteins in the dataset, we can generate a huge number, possibly multimillions, of similar peptides with localization annotation. Each record in the knowledge base is indexed by a similar peptide, and stores its similar peptide sequences and protein sources (those that are used as query proteins in the PSI-BLAST searches), similarity level and localization site information (inferred from the corresponding protein sources). Note that a similar peptide may occur multiple times in different HSPs of a single PSI-BLAST search result, i.e., derived from different similar proteins found in the PSI-BLAST search. We cluster them together and store the frequency in the peptide record. Table 1 shows a record of the similar peptide MYSKILL (assuming that the window size is 7), which is generated by performing PSI-BLAST search on the three proteins $(A, B$, and $C$ ) with known localization sites, respectively. The frequencies of MYSKILL in the PSI-BLAST search results of proteins $A, B$, and $C$ are 21 , 12 , and 17 , respectively. The localization site information is inherited from the three protein sources.

\section{KnowPred $_{\text {site: }}$ a localization prediction method using SPKB}

The main idea of KnowPred ${ }_{\text {site }}$ is illustrated in Figure 3. Given a target protein $t$, whose localization annotation is unknown and to be predicted, we perform PSI-BLAST search and use the same procedure as described in the last subsection for knowledge base construction to generate all similar peptides of $t$ and their frequencies from its native sequence and HSPs. Each similar peptide $h p$ is then matched against $S P K B$, and the peptide record with index $h p$ is called a hit. For each hit, we calculate two types of scores associated with each localization site

Table I: A similar peptide example

Similar Peptide: MYSKILL

\begin{tabular}{lllll}
\hline Protein Source & Localization Sites & Native Peptide Sequence & Similarity Level & Frequency \\
\hline A & Cytoplasm & MYKKILY & 5 & 21 \\
B & Nuclear & MYSSIL & 4 & 12 \\
C & Cytoplasm Extracellular & MYSSILY & 5 & 17 \\
\hline
\end{tabular}

Three protein sources with known localization sites contain peptides that are aligned and similar to the peptide MYSKILL in their HSPs. The similarity level indicates the number of amino acid pairs that are interchangeable between the native peptide sequence and the similar peptide sequence. The frequency represents the number of occurrences they are aligned in HSPs. 


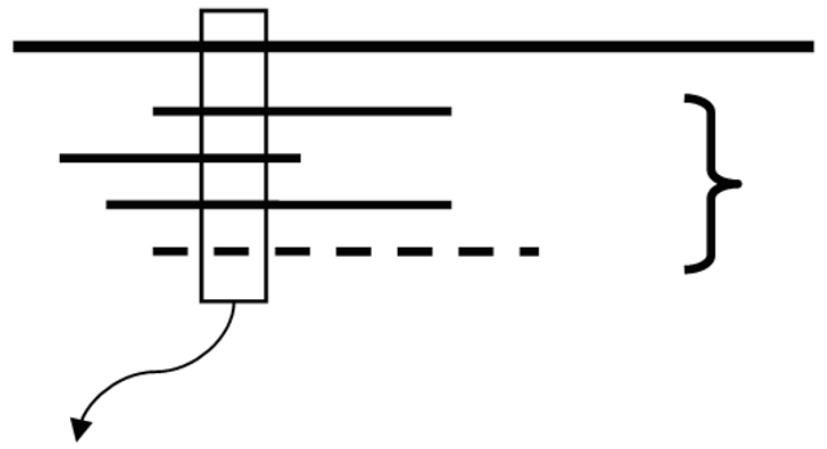

\title{
Query sequence of length $l$
}

\author{
Similar proteins found by
}

PSI-BLAST (HSPs)

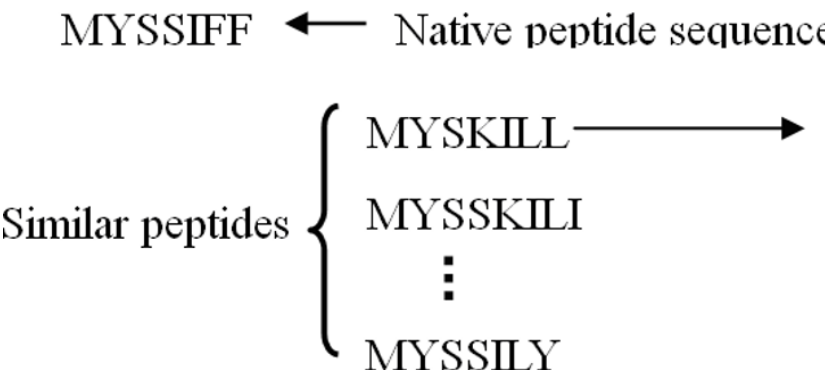

\begin{tabular}{|l|l|l|l|l|}
\hline Protein Source & Localization Sites & Peptide & Similarity Level & Frequency \\
\hline A & cytoplasm & MYKKILY & 5 & 21 \\
\hline B & nuclear & MYSSIIL & 4 & 12 \\
\hline C & cytoplasm, extracellular & MYSSILY & 5 & 17 \\
\hline
\end{tabular}

Similar peptide record in $S P K B$

Figure 3

The main algorithm of KnowPred site.

$i$ : the voting score $s_{i}$ and the confidence score CS $(i)$. The calculation of the voting score $s_{i}$ is as follows: Let $f$ denote the frequency of $h p$ found in all $t^{\prime}$ s HSPs. For each record in $S P K B$, we calculate the score $w_{i}$ associated with each localization site by summing up the frequencies of the similar peptides that contain the specific site. For example, for the peptide record MYSKILL shown in Table 1, the score of cytoplasm is $38(21+17$; since protein source $A$ and $C$ are both localized into cytoplasm), and those of nuclear and extracellular are 12 and 17, respectively. Then the voting score $s_{i}$ is defined as $f$ multiplied by $\left(w_{i} /\right.$ total frequencies in that record). For example, if MYSKILL is a similar peptide of $t$ and its frequency is 10 in $t^{\prime}$ s HSPs, then the voting scores of cytoplasm, nuclear, and extracellular are $7.6(=10 \times$ $38 / 50), 2.4(=10 \times 12 / 50)$, and $3.4(=10 \times 17 / 50)$, respectively, while those of other localization sites are all 0.

The localization site prediction of the protein $t$ is determined by the confidence score CS $(i)$, which is the total voting score aggregated from all hit records. Finally, each CS $(i)$ is divided by the summation of all frequencies $f$ of all $t^{\prime}$ s hits and then multiplied by 100 to normalize the confidence score in the range of 0 and 100. KnowPred ${ }_{\text {site }}$ predicts $t$ being localized into the site with the highest confidence score for single-localized proteins or into the sites with the two highest confidence scores for multi-localized proteins (All multi-localized proteins in ngLOC dataset have two localization sites).
To differentiate single-localized proteins from those that are multi-localized, we followed King and Guda's method [23] to calculate the multi-localized confidence score (MLCS) associated with a protein $t$, which gives a relative measure of the likelihood that the protein $t$ is multi-localized. It is derived from the two highest confidence scores (denoted as $C S_{1}$ and $C S_{2}$ ) and is defined as follows:

$$
\operatorname{MLCS}(t)=\left(C S_{1}+C S_{2}\right)-\frac{\left(C S_{1}^{2}-C S_{2}^{2}\right)}{100.0}
$$

and $\operatorname{MLCS}(t)$ is bounded by 100 , i.e., when the calculated MLCS $(t)$ is over 100, it is assigned 100 .

\section{BLAST-hit prediction method}

Since BLAST is the most popular method for sequence comparison, we implemented a simple prediction method based on the BLAST search result. Given a dataset of proteins with known localization site(s), to predict the localization site(s) of a test protein $t$ we first perform the BLAST search against the dataset and then assign the localization annotations of the best BLAST hit to the protein $t$. If there is no hit at the e-value cutoff 0.001 , no annotation will be assigned to the protein $t$. As reported by Jones and Swindells, the e-value of 0.001 generally produces a safe searching [28]. The performance of BLAST-based prediction method is usually 
treated as the baseline to compare with those of other methods [29].

\section{Evaluation measure}

The performance is estimated using the following measurements. To assess the performance in each localization site, precision, accuracy and Matthew's correlation coefficient (MCC) are calculated by Equations (1) to (3), respectively. The overall accuracy is defined in Equation (4).

$$
\begin{gathered}
\text { Precision }=\frac{T P_{i}}{T P_{i}+F P_{i}} \times 100 \% \\
\text { Accuracy }_{i}=\frac{T P_{i}}{N_{i}} \times 100 \% \\
\text { Accuracy }_{i}=\frac{T P_{i} \times T N_{i}-F P_{i} \times F N_{i}}{\sqrt{\left(T P_{i}+F P_{i}\right)\left(T P_{i}+F N_{i}\right)\left(T N_{i}+F P_{i}\right)\left(T N_{i}+F N_{i}\right)}} \\
\sum_{i=1}^{10} T P_{i} / \sum_{i=1}^{10} N_{i}
\end{gathered}
$$

where $T P_{i}, T N_{i}, F P_{i}, F N_{i}$ and $N_{i}$ are, respectively, the number of true positives, true negatives, false positives, false negatives, and proteins in localization site i. MCC, which considers both under- and over-predictions, provides a complementary measure of the predictive performance, where $M C C=1$ indicates a perfect prediction, $\mathrm{MCC}=0$ indicates a completely random assignment, and MCC = -1 indicates a perfectly reverse correlation.

\section{Results}

KnowPred $_{\text {site }}$ was implemented as a parallel program under the Linux environment. It was implemented using $\mathrm{C}++$ and MPICH library. We used the ngLOC dataset [23] to construct the knowledge base and test the performance of KnowPred site $_{\text {. The dataset is compiled }}$ from 1923 different species and contains 28056 protein sequences (listed in Additional file 1), including 25887 single localized proteins and 2169 multi-localized proteins. There are ten different subcellular locations among these proteins, which are Cytoplasm (CYT), Cytoskeleton (CSK), Endoplasmic Reticulum (END), Extracellular (EXC), Golgi Apparatus (GOL), Lysosome (LYS), Mitochondria (MIT), Nuclear (NUC), Plasma Membrane (PLA), and Perixosome (POX).

We conducted two types of experiment on the dataset. First, in order to take advantages of local similarities from as many proteins as possible, we conducted the leave-one-out cross validation experiment to determine the parameters and to evaluate the performance of

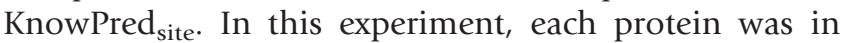
turn used as the test protein and the remaining 28055 proteins were used to construct the knowledge base. Second, we compared the performance of KnowPred ${ }_{\text {site }}$ with existing methods. Since the dataset is from ngLOC and ngLOC has been shown to be better than PSORT [30], pTARGET [31] and PLOC [32] using the same dataset, we directly compare KnowPred site $_{\text {against }}$ ngLOC using ten-fold cross validation. In this experiment, all proteins were partitioned into 10 subsets, and each subset was in turn used as the test set and the remaining nine subsets were used to construct the knowledge base.

\section{Determining window size $w$ and similarity threshold $\mathbf{k}$ for KnowPred site $_{\text {. }}$}

KnowPred $_{\text {site }}$ aims to utilize the localization annotations of similar peptides. The determination of similar relations, which depends on the window size $w$ and the threshold of similarity level $k$, can affect the

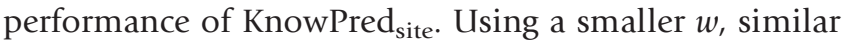
peptides have a higher probability to be hit against the knowledge base; however, shorter peptide sequences are likely to appear in many unrelated proteins. Given a fixed $w$, there is also a trade-off in choosing the threshold of similarity level $k$. A smaller $k$ produces looser similarity relations, which leads to extracting more, but less reliable, similar peptides. To make an appropriate selection of $w$ and $k$, we conducted a leave-one-out cross validation experiments on only the single-localized proteins in the ngLOC dataset for $w$ ranging from 3 to 11 and $k$ ranging from 0 to $w$.

Figure 4 shows the overall accuracies of KnowPred site $_{\text {. }}$ using different window size $w$ with fixed similarity threshold $(k=0)$. It shows that the appropriate window size is 7 or 8 . Then we further investigate the performance using different thresholds of similarity levels. Table 2 shows the overall accuracies ranging from $90.9 \%$ to $92.0 \%$ for all combinations of window sizes $(w=7,8)$ and similarity thresholds. According to the experiment results, we chose the combination of $w=7$ and $k=6$ for the following experiments since they provided the best accuracy $92.0 \%$.

\section{Prediction performance of KnowPred site}

After the best parameters have been determined, we conducted a ten-fold cross validation experiment on the entire dataset to compare KnowPred site $_{\text {with ngLOC and }}$ Blast-hit prediction. We used the top $N$ accuracy for evaluation, where $N$ ranges from 1 to 4 . A protein is 


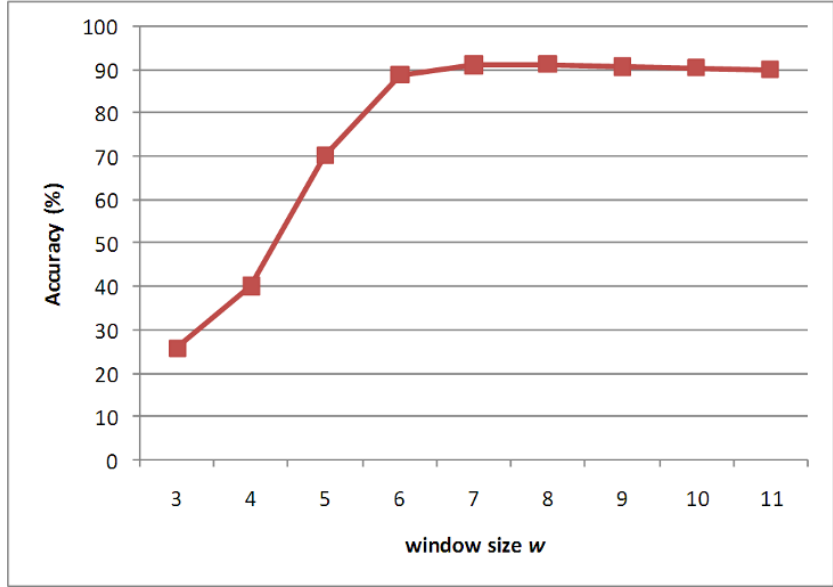

Figure 4

The overall accuracies of KnowPred site $_{\text {using }}$ different size of similar peptide length.

considered to be correctly predicted when the real localization site(s) rank among the top $N$ of the predicted sites. (Top 1 accuracy is simply the Accuracy defined in Equation (4).) Notably, for multi-localized proteins, the accuracy is measured in two ways: first, at least one site correctly predicted and second, both sites correctly predicted. Using the first measurement, a true positive is a multi-localized protein with at least one localization site correctly predicted; whereas a true positive using the second measurement is a multilocalized protein with both sites correctly predicted.

The prediction performance of KnowPred $_{\text {siter }}$ ngLOC, and Blast-hit is summarized in Table 3, in which KnowPred $_{\text {site }}$ performance is reported with ten-fold cross validation and leave-one-out cross validation as denoted by ${ }^{*}$ KnowPred $_{\text {site }}$ and ${ }^{*}$ KnowPred $_{\text {siter }}$ respectively. It is observed that KnowPred site $_{\text {outperforms }}$ ngLOC and Blast-hit. (The prediction results of singleand multi-localized proteins by KnowPred $_{\text {site }}$ can be found in Additional file 2 to Additional file 5. Additional file 2 lists the prediction results for single-localized proteins using leave-one-out cross validation; Additional file 3 lists the prediction results for single-localized proteins using ten-fold cross validation; Additional file 4 lists the prediction results for multi-localized proteins using leave-one-out cross validation; Additional file 5 lists the prediction results for multi-localized proteins using ten-fold cross validation.)

For single-localized proteins, the overall accuracies of KnowPred $_{\text {site }}$ are from 91.7 to 98.1 when the correct prediction is considered within the top 1 to top 4 most probable sites. Those of ngLOC are from $88.8 \%$ to $96.3 \%$. The accuracy of Blast-hit is $86.0 \%$, which means $86.0 \%$ of single-localized proteins could be correctly predicted by BLAST searches. It is noteworthy that 2114 sequences among all single-localized proteins failed to find significant similar proteins by Blast-hit method; however, $58.8 \%$ of them were correctly predicted by KnowPred $_{\text {site. It shows that the local similarity helps }}$ identify related sequences for subcellular localization prediction.

The experiment result shows that KnowPred $_{\text {site }}$ has much higher accuracy on multi-localized proteins than the other methods. Using the first accuracy measurement, i.e., at least one site correctly predicted, KnowPred $_{\text {site }}$ achieves more than $90 \%$ of the top 1 accuracy, which is higher than ngLOC by $8.2 \%$. Using the tighter second accuracy measurement, KnowPred site $_{\text {achieves }}$ $72.1 \%$ of the top 2 accuracy, which is higher than ngLOC by $12.4 \%$. Further observing the top $\mathrm{N}$ accuracy, we find that KnowPred $_{\text {site }}$ is more able to narrow down the number of false positives than ngLOC.

The top 1 and top 2 accuracies of the Blast-hit method are $78.8 \%$ and $45.7 \%$ for the two accuracy measurements. Notably, 318 proteins among all multi-localized proteins failed to find any significant Blast hit; however, $73.3 \%$ and $49.7 \%$ of them were correctly predicted by KnowPred $_{\text {site }}$ using the two accuracy measurements, respectively.

\section{Site-specific prediction performance}

In contrast to the overall accuracy of the dataset reported in Table 3, we further analyze the prediction performance on each of the 10 distinct localization sites. The

Table 2: The overall accuracies using different thresholds of similarity levels for window size 7 and 8

\begin{tabular}{|c|c|c|c|c|c|c|c|c|c|}
\hline Similarity Level Threshold $k$ & 0 & $\mathbf{I}$ & 2 & 3 & 4 & 5 & 6 & 7 & 8 \\
\hline $\begin{array}{l}\text { Overall Accuracy (\%) } \\
w=7\end{array}$ & 91.2 & 91.2 & 91.3 & 91.4 & 91.5 & 91.8 & 92.0 & 91.6 & - \\
\hline $\begin{array}{l}\text { Overall Accuracy (\%) } \\
w=8\end{array}$ & 91.4 & 91.4 & 91.4 & 91.4 & 91.4 & 91.5 & 91.6 & 91.7 & 90.9 \\
\hline
\end{tabular}

The combination of $w=7$ and $k=6$ provides the best accuracy. Some results are shown to have identical overall accuracies due to the rounding off to the first decimal place. 


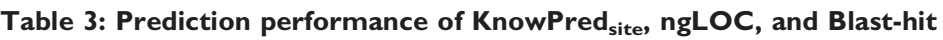

\begin{tabular}{|c|c|c|c|c|c|}
\hline Overall Accuracy (\%) & Methods & Top I & Top 2 & Top 3 & Top 4 \\
\hline \multirow[t]{4}{*}{ Single-localized } & ${ }^{* K n o w P r e d}$ site & 92.0 & 95.7 & 96.8 & 98.1 \\
\hline & ${ }^{\#}$ KnowPred $_{\text {site }}$ & 91.7 & 95.4 & 96.6 & 97.9 \\
\hline & ngLOC & 88.8 & 92.2 & 94.5 & 96.3 \\
\hline & Blast-hit & 86.0 & - & - & - \\
\hline \multirow{4}{*}{$\begin{array}{l}\text { Multi-localized } \\
\text { (at least I correct) }\end{array}$} & $*_{\text {KnowPred }}$ site & 90.8 & 96.4 & 98.2 & 98.9 \\
\hline & ${ }^{\#}$ KnowPred ${ }_{\text {site }}$ & 90.1 & 96.1 & 98.1 & 98.9 \\
\hline & ngLOC & 81.9 & 92.0 & 96.1 & 97.4 \\
\hline & Blast-hit & 78.8 & - & - & - \\
\hline \multirow{4}{*}{$\begin{array}{l}\text { Multi-localized } \\
\text { (both correct) }\end{array}$} & $*_{\text {KnowPred }}$ site & & 74.3 & 83.3 & 88.7 \\
\hline & ${ }^{\#}$ KnowPred ${ }_{\text {site }}$ & & 72.1 & 82.2 & 87.5 \\
\hline & ngLOC & & 59.7 & 73.8 & 83.2 \\
\hline & Blast-hit & & 45.7 & - & - \\
\hline
\end{tabular}

*KnowPredsite represents the experiment result using leave-one-out cross validation; \#KnowPredsite represents the experiment result using I0-fold cross validation.

results are summarized in Table 4. Among the 10 localization sites, the precision ranges from $75.7 \%$ to $98.5 \%$ and the accuracy $_{i}$ ranges from $52.0 \%$ to $96.4 \%$. It is observed that higher occurrence of the localization site, e.g., EXC (29.1\%) and PLA (25.2\%), leads to better prediction, e.g., the precision and accuracy on EXC are $98.5 \%$ and $93.9 \%$, respectively. Low occurrence of the localization site can deteriorate prediction, for example, CSK $(1 \%)$ and GOL (1.1\%) have MCC of 0.645 and 0.746 , respectively. However, if the similar peptide records of a site have higher specificity, prediction performance can be good despite low occurrence. For example, the precision and accuracy on LYS (0.6\%) and POX $(0.8 \%)$ are $87.2 \%$ and $81.9 \%$, and $87.3 \%$ and $85.1 \%$, respectively. Furthermore, it is noteworthy that although CYT represents $11.1 \%$ of the dataset, its $\mathrm{MCC}_{\mathrm{i}}$ is 0.774 , much lower than other highly occurring sites. Its low $\mathrm{MCC}_{\mathrm{i}}$ is due to low precision since KnowPredsite yields more false positives for CYT. High false positives usually occur when the similar peptide records of a site have lower specificity and higher diversity. As a result, proteins of other localization sites are misclassified as CYT.

Figure 5 shows the site-specific comparison between KnowPred $_{\text {site }}$ and ngLOC in terms of accuracy and MCC. KnowPred $_{\text {site }}$ outperforms ngLOC in eight localization sites (CSK, END, EXC, GOL, MIT, NUC, PLA, POX) in terms of MCC. The two sites where ngLOC performs better are CYT $(0.777$ for ngLOC and 0.774 for KnowPred $_{\text {site }}$ ) and LYS (0.902 for ngLOC and 0.844 for KnowPred $\left._{\text {site }}\right)$. In terms of accuracy, KnowPred ${ }_{\text {site }}$ outperforms ngLOC in all sites except for LYS (representing around $0.6 \%$ of the whole dataset), where ngLOC and KnowPred $_{\text {site }}$ yields $85.5 \%$ and $81.9 \%$ of accuracy, respectively.

\section{Evaluation of the multi-localized confidence score (MLCS)}

A significant number of eukaryotic proteins are known to be localized into multiple subcellular organelles;

Table 4: Prediction performance of KnowPred $_{\text {site }}$ for each site using precision, accuracy, and MCC

\begin{tabular}{|c|c|c|c|c|}
\hline Site $i$ & Occurrence in the dataset (\%) & Precision (\%) & Accuracy $_{i}(\%)$ & $M C C_{i}$ \\
\hline CYT & II.I & 75.7 & 84.4 & 0.774 \\
\hline CSK & 1.0 & 81.1 & 52.0 & 0.645 \\
\hline END & 3.6 & 92.9 & 84.1 & 0.88 \\
\hline EXC & 29.1 & 98.5 & 93.9 & 0.946 \\
\hline GOL & 1.1 & 79.1 & 70.9 & 0.746 \\
\hline LYS & 0.6 & 87.2 & 81.9 & 0.844 \\
\hline MIT & 9.4 & 96.7 & 86.9 & 0.907 \\
\hline NUC & 18.0 & 87.3 & 93.8 & 0.884 \\
\hline PLA & 25.2 & 94.4 & 96.4 & 0.938 \\
\hline POX & 0.8 & 87.3 & 85.1 & 0.861 \\
\hline
\end{tabular}




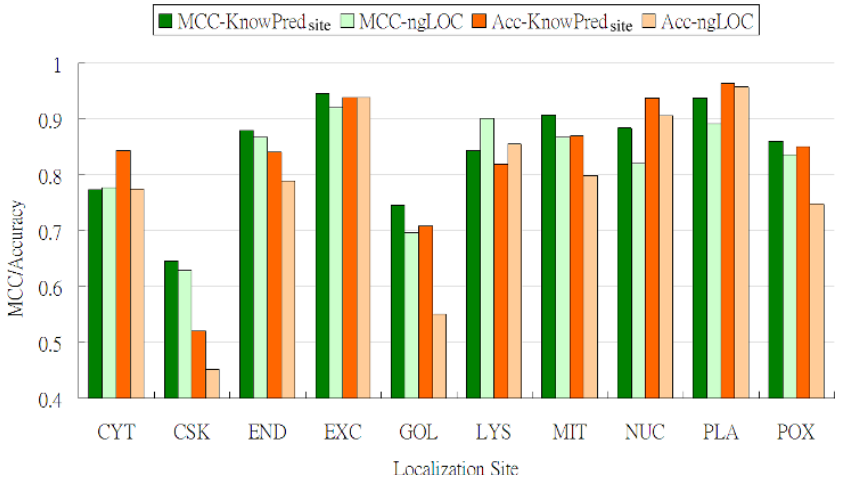

Figure 5

Matthew's correlation coefficient (MCC) and accuracy comparison between KnowPred site $_{\text {and }}$ ngLOC.

therefore, it is important to differentiate single-localized proteins from multi-localized proteins. We used the entire ngLOC dataset to compare different MLCS thresholds on the correct distinction between single-localized and multi-localized proteins. Specifically, we used the portions of true positives in the multi-localized proteins and true negatives in the single-localized proteins as the performance measures. A true positive represents a multi-localized protein whose MLCS is above the threshold and a true negative represents a singlelocalized protein whose MLCS is below the threshold.

We illustrate the cumulative percentages of true positive and true negative versus the MLCS threshold in Figure 6, which shows that the true negative curve is increasing along the MLCS axis whereas the true positive curve is decreasing. If the MLCS threshold is set to be 40,60.7\% of multi-localized proteins are true positives and 96.5\% of single-localized proteins are true negatives. It shows that $60.7 \%$ of multi-localized proteins obtained MLCS of 40 or better, whereas only $3.5 \%$ of single-localized proteins within this range. If the MLCS threshold is set to be $20,86.3 \%$ of multi-localized proteins are true positives and $82.8 \%$ of single-localized proteins are true negatives. In ngLOC, the best result shows that $76 \%$ of multi-localized proteins belong to true positives and $81 \%$ of single-localized proteins belong to true negatives when 40 of MLCS threshold is applied. The result shows that KnowPred $_{\text {site }}$ better differentiate multi-localized proteins from those that are single-localized.

\section{Discussion}

Unlike most machine learning methods that the parameters of the prediction models are not biologically interpretable, the prediction result of KnowPred $_{\text {site }}$ is

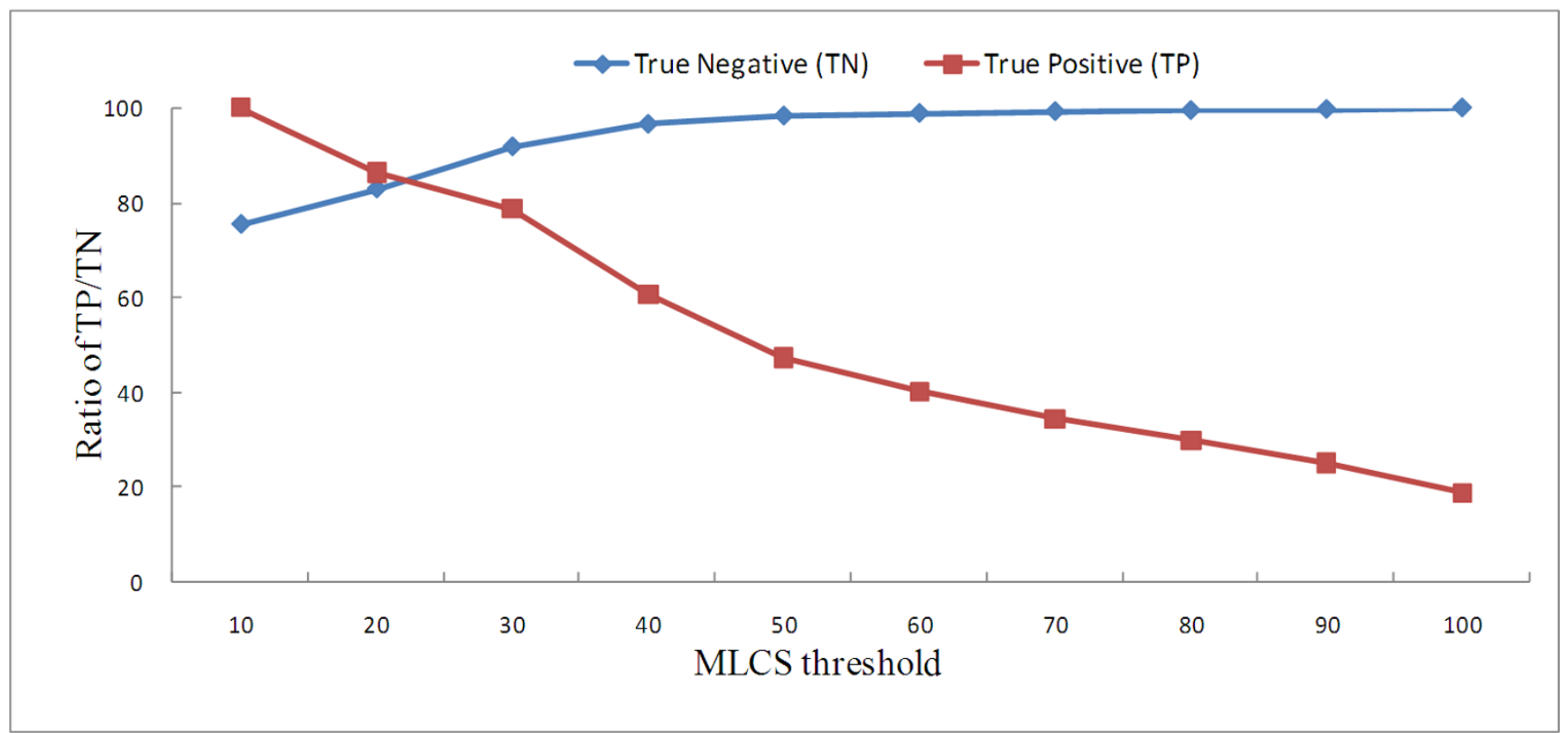

Figure 6

MLCS analysis. A true positive represents a multi-localized protein whose MLCS is above the threshold and a true negative represents a single-localized protein whose MLCS is below the threshold. We compare the ratio of true positives/true negatives to the total number of multi-/single-localized proteins. 
interpretable and the prediction process is transparent and traceable. To predict the localization sites of a protein, KnowPred ${ }_{\text {site }}$ shows the template sequences and their associated contributive confidence scores for a query protein. Such information is useful for interpretation of the prediction results. In this section, we select the four sequences EF1A2_RABIT, RASH_HUMAN, MCA3_MOUSE, and CFDP2_BOVIN from the ngLOC dataset, to demonstrate the interpretation of KnowPredsite prediction results.

The prediction result of each of the first three proteins and its template sequences extracted from the knowledge base used for prediction are shown in Table 5, 6, 7, respectively. In each table, the prediction result shows the MLCS and the confidence score of each localization site that the query protein would be localized into. Moreover, the template proteins which are used to vote for the localization sites are shown in each table. We only list the top eight template proteins which contribute most to the confidence scores of the query sequence. For each template sequence, its contribution to confidence score of each localization site and the sequence identity to the query protein calculated by ClustalW (denoted by SI) are shown.

In the example of EF1A2_RABIT shown in Table 5, KnowPred $_{\text {site }}$ predicts it being single-localized at cytoplasm (CYT) since MLCS is very low (7.40) and CYT has the highest confidence score. However, the localization site of EF1A2_RABIT reported in the ngLOC dataset is nuclear (NUC). Examining the eight template proteins, we find that they all have high sequence identities with EF1A2_RABIT and most of them are localized into CYT except EF1A2_RAT localized into NUC. According to the Gene Ontology annotation, it is localized into CYT and NUC, which are the two sites with the highest confidence

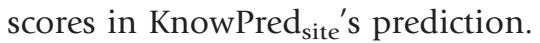

Table 5: Prediction result of EFIA2_RABIT

\begin{tabular}{lccccccccccc}
\hline Query & CYT & CSK & END & EXC & GOL & LYS & MIT & NUC* & PLA & POX & MLCS \\
\hline EFIA2_RABIT & 95.45 & 0 & 0 & 1.45 & 0 & 0 & 0.04 & 2.97 & 0.05 & 0 & 7.40 \\
\hline
\end{tabular}

\begin{tabular}{|c|c|c|c|c|c|c|c|c|c|c|c|}
\hline Template & CYT & CSK & END & EXC & GOL & LYS & MIT & NUC & PLA & POX & $\mathrm{SI}$ \\
\hline EFIA2_RAT & 0 & 0 & 0 & 0 & 0 & 0 & 0 & 2.94 & 0 & 0 & 99.78 \\
\hline EFIA_C $\mathrm{CHICK}$ & 2.77 & 0 & 0 & 0 & 0 & 0 & 0 & 0 & 0 & 0 & 92.22 \\
\hline EFIAI_HUMAN & 2.75 & 0 & 0 & 0 & 0 & 0 & 0 & 0 & 0 & 0 & 92.22 \\
\hline EFIAI_RAT & 2.75 & 0 & 0 & 0 & 0 & 0 & 0 & 0 & 0 & 0 & 92.22 \\
\hline EFIAO_XENLA & 2.69 & 0 & 0 & 0 & 0 & 0 & 0 & 0 & 0 & 0 & 90.06 \\
\hline EFIA_BRARE & 2.64 & 0 & 0 & 0 & 0 & 0 & 0 & 0 & 0 & 0 & 90.06 \\
\hline EFIAㄹ﹎_XENLA & 2.64 & 0 & 0 & 0 & 0 & 0 & 0 & 0 & 0 & 0 & 88.79 \\
\hline EFIA3_XENLA & 2.60 & 0 & 0 & 0 & 0 & 0 & 0 & 0 & 0 & 0 & 88.55 \\
\hline
\end{tabular}

*: correct answer; SI: sequence identity.

Table 6: Prediction result of RASH_HUMAN

\begin{tabular}{lccccccccccc}
\hline Query & CYT* & CSK & END & EXC & GOL* & LYS & MIT & NUC & PLA & POX & MLCS \\
\hline RASH_HUMAN & 18.95 & 0.06 & 0.09 & 0.09 & 13.74 & 0.04 & 0.24 & 0.25 & 83.61 & 0 & 36.24 \\
\hline
\end{tabular}

\begin{tabular}{|c|c|c|c|c|c|c|c|c|c|c|c|}
\hline Template & $\mathrm{CYT}$ & CSK & END & EXC & GOL & LYS & MIT & NUC & PLA & POX & SI \\
\hline RASK_HUMAN & 0 & 0 & 0 & 0 & 0 & 0 & 0 & 0 & 13.88 & 0 & 86.32 \\
\hline RASK_MOUSE & 0 & 0 & 0 & 0 & 0 & 0 & 0 & 0 & $13.8 \mid$ & 0 & 86.32 \\
\hline RASN_HUMAN & 13.19 & 0 & 0 & 0 & 13.19 & 0 & 0 & 0 & 0 & 0 & 85.19 \\
\hline LET60_CAEEL & 0 & 0 & 0 & 0 & 0 & 0 & 0 & 0 & 10.55 & 0 & 74.07 \\
\hline RAS3_RHIRA & 0 & 0 & 0 & 0 & 0 & 0 & 0 & 0 & 5.05 & 0 & 57.07 \\
\hline RASI_RHIRA & 0 & 0 & 0 & 0 & 0 & 0 & 0 & 0 & 4.88 & 0 & 58.62 \\
\hline RAS2_RHIRA & 0 & 0 & 0 & 0 & 0 & 0 & 0 & 0 & 4.33 & 0 & 35.20 \\
\hline RAS_LIMLI & 0 & 0 & 0 & 0 & 0 & 0 & 0 & 0 & 4.15 & 0 & 46.03 \\
\hline
\end{tabular}

*: correct answer; SI: sequence identity. 
Table 7: Prediction result of MCA3_MOUSE. Templates marked with ' + ' are those that have the same localization annotation with the query protein

\begin{tabular}{lccccccccccc}
\hline Query & CYT* & CSK & END & EXC & GOL & LYS & MIT & NUC* & PLA & POX & MLCS \\
\hline MCA3_MOUSE & 95.46 & 0.3 & 0.27 & 0.36 & 0.2 & 0.01 & 1.13 & 93.59 & 1.82 & 0.22 & 100 \\
\hline
\end{tabular}

\begin{tabular}{lccccccccccc}
\hline Template & CYT & CSK & END & EXC & GOL & LYS & MIT & NUC & PLA & POX & SI \\
\hline MCA3_HUMAN $^{+}$ & 89.16 & 0 & 0 & 0 & 0 & 0 & 0 & 89.16 & 0 & 0 & 88.51 \\
EFIGI_YEAST $^{+}$ & 2.74 & 0 & 0 & 0 & 0 & 0 & 0 & 2.47 & 0 & 0 & 8.67 \\
EFIG2_YEAST $_{\text {GSTA_PLEPL }}^{0.49}$ & 0.35 & 0 & 0 & 0 & 0 & 0 & 0.49 & 0 & 0 & 0 & 8.50 \\
SYEC_YEAST & 0.16 & 0 & 0 & 0 & 0 & 0 & 0 & 0 & 0 & 0 & 15.86 \\
CCNAI_MOUSE & 0 & 0.15 & 0 & 0 & 0 & 0 & 0 & 0 & 0 & 0 & 3.86 \\
NUI55_RAT & 0.14 & 0 & 0 & 0 & 0 & 0 & 0 & 0.14 & 0 & 0 & 7.36 \\
GCYB2_HUMAN $^{+}$ & 0.14 & 0 & 0 & 0 & 0 & 0 & 0 & 0 & 0 & 0 & 4.17 \\
\hline
\end{tabular}

*: correct answer; SI: sequence identity.

In the example of RASH_HUMAN shown in Table 6, KnowPred $_{\text {site }}$ predicts RASH_HUMAN being localized into plasma membrane (PLA) and cytoplasm (CYT). However, the correct localization site is cytoplasm and Golgi apparatus (GOL). Referring to the prediction result, the confidence score of PLA is much higher than those of CYT and GOL. It is also observed that most of the template proteins are localized into PLA. According to the annotation in Gene Ontology and SwissProt, RASH_HUMAN is localized into PLA and GOL, and the template protein, RASN_HUMAN, is also localized into PLA and GOL. If applying the new annotation data, KnowPred $_{\text {site }}$ can predict RASH_HUMAN correctly.

As for MCA3_MOUSE shown in Table 7, KnowPred site predicts its MLCS 100 and it being localized into cytoplasm (CYT) and nuclear (NUC) correctly. Examining the template proteins, we observe that KnowPred $\mathrm{d}_{\text {site }}$ identifies some related proteins, i.e., which have the same localization with the query protein. EF1G1_YEAST and NU155_RAT, even though they share very low sequence identity $8.67 \%$ and $3.17 \%$, respectively, with the query protein. Notably, the two template proteins rank second and seventh, respectively, among all template proteins. Furthermore, though GSTA_PLEPL has higher sequence identity $(15.86 \%)$ with the query protein than EF1G1_YEAST, the confidence score contributed by EF1G1_YEAST is much higher than that by GSTA_PLEPL (2.74 vs. 0.35). It shows that the contributive confidence score is not necessary to be positively correlated with the sequence identity when template sequences are dissimilar with the query sequence. In this example, EF1G1_YEAST shares more local similarities (peptide fragments) with the query protein than GSTA_PLEPL does. If MCA3_HUMAN, the one that shares $88.51 \%$ sequence identity with the query protein, is taken out from the template pool,
KnowPred $_{\text {site }}$ can still predict correctly for protein MCA3_MOUSE.

For the multi-localized proteins, there are 318 proteins unable to find similar sequences by the Blast-hit method. However, the localization sites of around half of them can be correctly predicted by KnowPred $\mathrm{H}_{\text {site. }}$ We randomly choose an example, CFDP2_BOVIN, to demonstrate the KnowPred $_{\text {site's capability of identifying related sequences }}$ from the template pool. The two highest confidence scores of CFDP2_BOVIN are 32.07 (CYT) and 41.18 (NUC). Among the top 100 templates (ranked by the contribution to the confidence scores), 12 of them are localized into CYT and NUC, 18 are localized into CYT only, and 32 are localized into NUC only. Their sequence identities against CFDP2_BOVIN are very low, ranging from $3.47 \%$ to $13.8 \%$. The result suggests that local similarity captured by our method is beneficial for PSL prediction when global sequence similarity is very low.

\section{Conclusion}

In this paper, we propose a highly accurate subcellular localization prediction method for single- and multilocalized proteins, called KnowPred siter $_{\text {, which is based }}$ on a knowledge base instead of frequently used machine learning approaches. The knowledge base, called SPKB, is constructed from a given dataset of proteins with known localization site annotation to capture local similarity between proteins so that related proteins with the same localization can be identified. Using these related proteins obtained form the knowledge base, the localization site of a query protein can be better predicted.

We used the ngLOC dataset to evaluate the performance of KnowPred $_{\text {site. }}$ The dataset consists of 25887 single-localized 
proteins and 2169 multi-localized proteins of ten subcellular proteomes from 1923 species. In order to compare KnowPred $_{\text {site }}$ with ngLOC and the baseline Blast-hit method, we performed ten-fold cross validation on the dataset. The experiment results show that KnowPred ${ }_{\text {site }}$ achieves higher prediction accuracy than ngLOC and Blast-hit. Particularly, on multi-localized sequences KnowPred ${ }_{\text {site }}$ outperformed ngLOC by $8.2 \%$ in accuracy when a protein is correctly predicted if at least one site is correctly identified and by $12.4 \%$ in accuracy when a protein is correctly predicted if both sites are correctly identified.

A major advantage of knowledge base approaches is that the prediction process is transparent and interpretable. We can examine the prediction process to see how KnowPred $_{\text {site }}$ generates the prediction. Furthermore, with close observation from the prediction results in our experiments as described in the Discussion section, we find that KnowPred ${ }_{\text {site }}$ can efficiently use local similarity to identify related sequences even when their sequence identity is low so as to predict localization site with high accuracy.

When more proteins have known localization sites, most machine learning based methods need to retrain the prediction models, In contrast, KnowPred $\mathrm{H}_{\text {site }}$ can be easily improved by incrementally expanding the knowledge base, i.e., adding new peptide records or updating existing records with new protein sources and their localization site information. This feature indicates the expansibility and efficiency in maintaining the KnowPred $_{\text {site }}$ prediction system.

\section{Competing interests}

The authors declare that they have no competing interests.

\section{Authors' contributions}

Hsin-Nan Lin developed the method, carried out the computational predictions. Ching-Tai Chen and HsinNan Lin were involved in the literature survey, result interpretation, statistical analysis, and manuscript writing. Ting-Yi Sung, Shinn-Ying Ho and Wen-Lian Hsu coordinated the study and revised the manuscript. All authors read and approved the final manuscript.

\section{Note}

Other papers from the meeting have been published as part of BMC Genomics Volume 10 Supplement 3, 2009: Eighth International Conference on Bioinformatics (InCoB2009): Computational Biology, available online at http://www. biomedcentral.com/1471-2164/10?issue=S3.

\section{Additional material}

\author{
Additional file 1 \\ ngLOC dataset. The file contains whole ngLOC dataset, in which the \\ row starts with '>' represents the protein name, the next row represents \\ localization site. The localization site is numbered from 1 to 10 , \\ denoting Cytoplasm (CYT), Cytoskeleton (CSK), Endoplasmic \\ Reticulum (END), Extracellular (EXC), Golgi Apparatus (GOL), \\ Lysosome (LYS), Mitochondria (MIT), Nuclear (NUC), Plasma \\ Membrane (PLA), and Perixosome (POX). The ngLOC dataset can be \\ also downloaded via http://bio-cluster.iis.sinica.edu.tw/kbloc/DataSet. \\ htm. \\ Click here for file \\ [http://www.biomedcentral.com/content/supplementary/1471- \\ 2105-10-S15-S8-S1.txt]
}

\section{Additional file 2}

KnowPred $_{\text {site }}$ prediction results for single-localized proteins using leave-one-out cross validation. Each row is a prediction result for a protein sequence. Columns $A$, and $B$ represent protein name and localization site annotation, respectively. Columns $C$ to $L$ are the confidence scores corresponding to each localization site. Columns $N$ to $Q$ are the Top 1 to Top 4 accuracies.

Click here for file

[http://www.biomedcentral.com/content/supplementary/14712105-10-S15-S8-S2.csv]

\section{Additional file 3}

KnowPred $_{\text {site }}$ prediction results for single-localized proteins using ten-fold cross validation. The columns' definition is the same as that for Additional File 2.

Click here for file

[http://www.biomedcentral.com/content/supplementary/14712105-10-S15-S8-S3.csv]

\section{Additional file 4}

KnowPred $_{\text {site }}$ prediction results for multi-localized proteins using leave-one-out cross validation. Each row is a prediction result for a protein sequence. Columns A to L are the same to Additional File 2. Columns $N$ to $Q$ are the Top 1 to Top 4 accuracies based on the "at least one correct" criterion. Columns $S$ to $U$ are Top 2 to Top 4 accuracies based on the "both correct" criterion.

Click here for file

[http://www.biomedcentral.com/content/supplementary/14712105-10-S15-S8-S4.csv]

\section{Additional file 5}

KnowPred $_{\text {site }}$ prediction results for multi-localized proteins using tenfold cross validation. The columns' definition is the same as that for Additional file 4.

Click here for file

[http://www.biomedcentral.com/content/supplementary/14712105-10-S15-S8-S5.csv]

\section{Acknowledgements}

Thanks to all who developed PSI-BLAST and made it publicly available. We also appreciate Chia-Yu Su for helpful discussions.

This article has been published as part of BMC Bioinformatics Volume 10 Supplement 15, 2009: Eighth International Conference on Bioinformatics 
(InCoB2009): Bioinformatics. The full contents of the supplement are available online at http://www.biomedcentral.com//47/-2/05/I0? issue $=\mathrm{S} 15$.

\section{References}

I. Nair R and Rost B: Better prediction of sub-cellular localization by combining evolutionary and structural information. Proteins 2003, 53(4):917-930.

2. Gardy JL, Laird MR, Chen F, Rey S, Walsh CJ, Ester M and Brinkman FS: PSORTb v.2.0: expanded prediction of bacterial protein subcellular localization and insights gained from comparative proteome analysis. Bioinformatics 2005, 21(5):617-623.

3. Hoglund A, Donnes P, Blum T, Adolph HW and Kohlbacher O: MultiLoc: prediction of protein subcellular localization using $\mathrm{N}$-terminal targeting sequences, sequence motifs and amino acid composition. Bioinformatics 2006, 22(10): I I58-1 I65.

4. Wang JR, Sung WK, Krishnan A and Li KB: Protein subcellular localization prediction for Gram-negative bacteria using amino acid subalphabets and a combination of multiple support vector machines. BMC Bioinformatics 2005, 6:174.

5. $\mathrm{Yu}$ CS, Chen YC, Lu CH and Hwang JK: Prediction of protein subcellular localization. Proteins 2006, 64(3):643-65I.

6. Yu CS, Lin CJ and Hwang JK: Predicting subcellular localization of proteins for Gram-negative bacteria by support vector machines based on n-peptide compositions. Protein Sci 2004, 13(5): | 402-1406.

7. Chang JM, Su EC, Lo A, Chiu HS, Sung TY and Hsu WL: PSLDoc: Protein subcellular localization prediction based on gappeddipeptides and probabilistic latent semantic analysis. Proteins 2008, 72(2):693-7I0.

8. Bhasin M, Garg A and Raghava GP: PSLpred: prediction of subcellular localization of bacterial proteins. Bioinformatics 2005, 2 I (I0):2522-2524.

9. Chou KC and Cai YD: Predicting protein localization in budding yeast. Bioinformatics 2005, 21 (7):944-950.

10. Gardy JL, Spencer C, Wang K, Ester M, Tusnady GE, Simon I, Hua S, deFays K, Lambert C and Nakai K, et al: PSORT-B: Improving protein subcellular localization prediction for Gram-negative bacteria. Nucleic Acids Res 2003, 3I(13):36I3-36I7.

II. Lee K, Kim DW, Na D, Lee KH and Lee D: PLPD: reliable protein localization prediction from imbalanced and overlapped datasets. Nucleic Acids Res 2006, 34(I7):4655-4666.

12. Nair R and Rost B: Mimicking cellular sorting improves prediction of subcellular localization. I Mol Biol 2005, 348 (I):85-100.

13. Huang WL, Tung CW, Ho SW, Hwang SF and Ho SY: ProLoc-GO: utilizing informative Gene Ontology terms for sequencebased prediction of protein subcellular localization. BMC Bioinformatics 2008, 9:80.

14. Marcotte EM, Xenarios I, Bliek van Der AM and Eisenberg D: Localizing proteins in the cell from their phylogenetic profiles. Proc Natl Acad Sci USA 2000, 97(22): 12115-12120.

15. Mott R, Schultz J, Bork $P$ and Ponting CP. Predicting protein cellular localization using a domain projection method. Genome Res 2002, I2(8): I |68-1 I74.

16. Su EC, Chiu HS, Lo A, Hwang JK, Sung TY and Hsu WL: Protein subcellular localization prediction based on compartmentspecific features and structure conservation. BMC Bioinformatics 2007, 8:330.

17. Rychlewski L, Jaroszewski L, Li WZ and Godzik A: Comparison of sequence profiles. Strategies for structural predictions using sequence information. Protein Science 2000, 9(2):232-24I.

18. Sadreyev R and Grishin N: COMPASS: A tool for comparison of multiple protein alignments with assessment of statistical significance. Journal of Molecular Biology 2003, 326(I):317-336.

19. Przybylski $D$ and Rost $B$ : Consensus sequences improve PSIBLAST through mimicking profile-profile alignments. Nucleic Acids Research 2007, 35(7):2238-2246.

20. Pietrokovski S: Searching databases of conserved sequence regions by aligning protein multiple-alignments. Nucleic Acids Research 1996, 24(19):3836-3845.

21. Yona $G$ and Levitt $M$ : Within the twilight zone: A sensitive profile-profile comparison tool based on information theory. Journal of Molecular Biology 2002, 3 I5(5): I 257-I275.

22. Zhang S, Xia X, Shen J, Zhou Y and Sun Z: DBMLoc: a Database of proteins with multiple subcellular localizations. $B M C$ Bioinformatics 2008, 9:127.
23. King BR and Guda C: ngLOC: an n-gram-based Bayesian method for estimating the subcellular proteomes of eukaryotes. Genome Biology 2007, 8(5):

24. Lin HN, Chang JM, Wu KP, Sung TY and Hsu WL: HYPROSP II-a knowledge-based hybrid method for protein secondary structure prediction based on local prediction confidence. Bioinformatics 2005, 2I( I5):3227-3233.

25. Wu KP, Lin HN, Chang JM, Sung TY and Hsu WL: HYPROSP: a hybrid protein secondary structure prediction algorithm-a knowledgebased approach. Nucleic Acids Res 2004, 32(I7):5059-5065.

26. Chen CT, Lin HN, Sung TY and Hsu WL: HYPLOSP: a knowledge-based approach to protein local structure prediction. J Bioinform Comput Biol 2006, 4(6): | 287-| 307.

27. Bolten E, Schliep A, Schneckener S, Schomburg D and Schrader R: Clustering protein sequences-structure prediction by transitive homology. Bioinformatics 200 I, I7(10):935-94I.

28. Jones DT and Swindells MB: Getting the most from PSI-BLAST. Trends in Biochemical Sciences 2002, 27(3): 16I-164.

29. Forslund $\mathrm{K}$ and Sonnhammer ELL: Predicting protein function from domain content. Bioinformatics 2008, 24(15):|68|-|687.

30. Nakai $K$ and Horton P: PSORT: a program for detecting sorting signals in proteins and predicting their subcellular localization. Trends Biochem Sci 1999, 24(I):34-36.

3I. Guda C and Subramaniam S: pTARGET: a new method for predicting protein subcellular localization in eukaryotes. Bioinformatics 2005, 2 I(24):4434-4434.

32. Park KJ and Kanehisa M: Prediction of protein subcellular locations by support vector machines using compositions of amino acids and amino acid pairs. Bioinformatics 2003, I9(13):1656-1663.

Publish with BioMed Central and every scientist can read your work free of charge

"BioMed Central will be the most significant development for disseminating the results of biomedical research in our lifetime. "

Sir Paul Nurse, Cancer Research UK

Your research papers will be:

- available free of charge to the entire biomedical community

- peer reviewed and published immediately upon acceptance

- cited in PubMed and archived on PubMed Central

- yours - you keep the copyright
BioMedcentral 